\title{
Equine fetal adrenal, gonadal and placental steroidogenesis
}

\author{
Erin L Legacki ${ }^{1}$, Barry A Bal ${ }^{2}$, C Jo Corbin ${ }^{1}$, Shavahn C Loux², Kirsten E Scoggin², \\ Scott D Stanley ${ }^{3}$, and Alan J Conley ${ }^{1}$
}

${ }^{1}$ Department of Population Health and Reproduction, School of Veterinary Medicine, University of California, Davis California, USA, ${ }^{2}$ Gluck Equine Research Center, Department of Veterinary Science, University of Kentucky, Lexington Kentucky, USA, and ${ }^{3}$ Department of Molecular Biosciences, School of Veterinary Medicine, University of California, Davis California, USA

Correspondence should be addressed to A J Conley; Email: ajconley@ucdavis.edu

The authors apologize for errors in Figure 6 of their article published in the October 2017 issue of Reproduction (vol 154 iss 4 pp 445-454). The authors explain that the addition of data (Figure 6) on steroid concentrations in the chorioallantois to their manuscript on fetal adrenal and fetal gonadal steroids during development of the equine fetus was made in response to reviewer comments. However, in compiling, summarizing and graphing the data, the wrong units were used in the final figure. The manuscript as published represents the data in Figure 6 as "ng/g", when in fact they are "nmol/g". The authors very much regret having made the mistake and sincerely apologize for any confusion this might have caused.

Figure 6 is published in full below along with the corresponding legend.

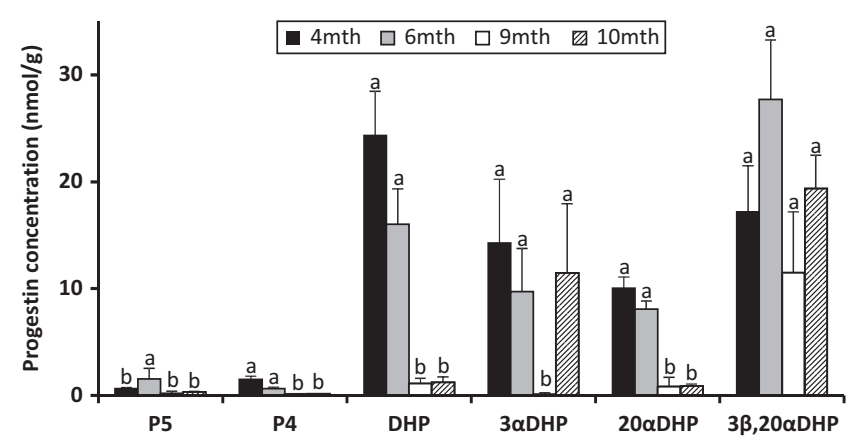

Figure 6 Equine allantochorionic concentrations of pregnenolone (P5), progesterone (P4) and $5 \alpha$-reduced pregnanes (DHP, dihydroprogesterone; $3 \alpha \mathrm{DHP}$, allopregnanolone; 20 $\alpha \mathrm{DHP}, 20 \alpha-$ hydroxy-DHP; $3 \beta, 20 \alpha \mathrm{DHP}, 3 \beta, 20 \alpha$-dihydroxyDHP; nmol/g) measured by LC-MS/MS in tissue at 4-10 months of gestation (mth). Comparisons among means for each steroid among gestational ages are as indicated by superscripts $\left({ }^{a, b}\right)$ where means with different superscripts differ, $P<0.05$. 